\title{
Introduction to magnetism
}

\author{
Laurent Ranno
}

Univ. Grenoble Alpes/CNRS, Institut Néel, 38042 Grenoble, France

\begin{abstract}
This text corresponds to an introductory lecture given at "Journées de la Neutronique" in May 2012 in Seignosse. It is meant as a short reminder about magnetism from its microscopic origins to its applications in many materials and devices which surround us. Only electronic magnetism is treated.
\end{abstract}

\section{INTRODUCTION}

Electromagnetism is one of the keystones of physics and even if we restrict ourselves to magnetism, it still represents a wide field of present day research in physics, material science and engineering. Research in magnetism is driven by the large impact it has on many aspects of everyday life, from electrical motors to data recording. On the one hand, many fundamental problems are still very active research fields and on the other hand, magnetism is closely related to material science.

Huge efforts are devoted to designing new model materials and improving existing materials by deeper understanding of the fundamental origins of their properties, keeping always in mind a possible feedback on their design. Permanent magnets which are sources of magnetic fields or store non volatile information, soft ferromagnets which guide, shield or sense magnetic fields are examples of the many use of magnetic materials. For both types of materials, the development of rare-earth - transition metals intermetallics system such as $\mathrm{NdFeB}$ or the fabrication of nanocrystalline ultrasoft magnetic materials has lead to breakthroughs in many applications in the last decade. Mastering the fabrication of magnetic structures at the characteristic lengthscale of spin transport or magnetic interaction has opened wide new fields in spintronics and micro-nanomagnetism. New effects can be evidenced (and so discovered) when materials are designed at the relevant lengthscale. Thanks to atomic layer deposition tools and self organization of particles, controlling nanometric thicknesses and sizes has become possible in the last decades but for many systems, reaching the relevant scale is still an open question. How to associate an internal controlled nanostructure and an external macroscopic size which can reach centimeters?

The vast range of possible magnetic materials, conducting or insulating, soft or hard, ferromagnetic/antiferromagnetic or paramagnetic and the large range of effects (from stray fields to order of magnitude choice of susceptibilities, induction, magnetotresistance, superparamagnetism...) makes certain that magnetism will be a fascinating and fruitful playground for magneticians for many more decades. 


\section{Collection SFN}

\section{MICROSCOPIC ORIGIN OF ATOMIC MAGNETISM}

The quantum nature of electrons makes spin and magnetism intrinsic ingredients of all electronic systems, including quantum fluids such as superconducting or superfluid states. Since electrons are not the only particles in materials, nuclear magnetism and its well known consequence: nuclear magnetic resonance (NMR) or magnetic resonance imaging (MRI) are also magnetism research fields. In this short text, we will restrict ourselves to electronic magnetism at high temperature (i.e. above $4 \mathrm{~K}$ !) and we will forget about quantum effects which appear at lower temperatures. First we will have a quick reminder about magnetic properties of isolated atoms and in a second part we will look at the impact of interactions to finish with a few words on materials.

\subsection{Quantum origins}

Electrons are elementary particles which are fermions. They must obey Fermi-Dirac statistics, which leads to the Pauli exclusion principle. The magnetic properties of atoms have two possible origins. The orbital motion of electrons gives rise to an orbital magnetic moment and their fermionic nature requires the existence of a half-integer spin ( $\frac{1}{2}$ for an electron), which is associated with a spin magnetic moment.

\subsection{Orbital magnetism}

In a classical picture, one electron on its atomic orbit is equivalent to a current loop (area A) i.e. a singleturn coil. Taking $r$ as the orbit radius, $v$ as the electron velocity, and $\vec{n}$ the unit vector perpendicular to the oriented orbit, the electron orbital magnetic moment $\vec{\mu}_{\text {orb }}$ is:

$$
\vec{\mu}_{o r b}=A \cdot I \vec{n}=\pi r^{2} \frac{-e v}{2 \pi r} \vec{n}=-\frac{r v e}{2} \vec{n} .
$$

One can also define its angular momentum $\vec{\sigma}$, with $m$ the electron mass:

$$
\vec{\sigma}=\vec{r} \wedge \vec{p}=\vec{r} \wedge m \vec{v}=m r v \vec{n} .
$$

Comparing magnetic and angular momenta, one can notice that they are proportional:

$$
\vec{\mu}_{\text {orb }}=-\frac{e}{2 m} \vec{\sigma}_{l}
$$

A proper quantum treatment of the problem does not change these results but the orbital momentum becomes an operator with quantised expectation values. $\vec{L}=\vec{r} \wedge \vec{p}$ becomes $L_{z}|\psi\rangle=l \hbar|\psi\rangle$ and $L^{2}|\psi\rangle=l(l+1) \hbar^{2}|\psi\rangle$.

So the angular momentum is quantized with $l$ defining its orbital quantum number ( $l$ is an integer):

$$
\vec{\sigma}_{l}=\hbar \vec{l} .
$$

As a direct consequence, the electron orbital magnetic moment is also quantized:

$$
\vec{\mu}_{l}=-\frac{e \hbar}{2 m} \vec{l}=-\mu_{B} \vec{l}
$$

$\mu_{B}$ is the Bohr magneton. It is the smallest possible value for an electron magnetic moment. It is the relevant order of magnitude to measure atomic magnetic moments and is often used as a unit for atomic magnetism.

$$
\Rightarrow 1 \mu_{B}=9.2710^{-24} \mathrm{Am}^{2}
$$




\subsection{Spin magnetism}

Besides its orbital moment which has a classical equivalent, an electron has also a spin moment which has a pure quantum origin. A possible image is that of a spinning top with two polarities for the spinning direction: $+\frac{1}{2}$ and $-\frac{1}{2}$. For an electron (spin $1 / 2$ fermion ) it gives:

$$
S_{z}|\psi\rangle= \pm \frac{1}{2} \hbar|\psi\rangle
$$

The spin angular momentum can be written as $\vec{\sigma}_{s}=\hbar \vec{s}$, with $s$ its spin. The spin magnetic moment associated with the spin angular momentum is $\vec{\mu}_{s}=-2 \mu_{B} \vec{s}$.

The total electron angular momentum is $\vec{\sigma}=\vec{\sigma}_{l}+\vec{\sigma}_{s}$ and its magnetic moment is $\vec{\mu}=-\mu_{B} \vec{l}-$ $2 \mu_{B} \vec{s}=-\frac{\mu_{B}}{\hbar}\left(\vec{\sigma}_{l}+2 \vec{\sigma}_{s}\right)$. So one can notice that the total angular momentum and the total magnetic moment are not simply proportional. Note also that magnetic moment and angular moment are antiparallel.

\subsection{Spin-orbit coupling}

The Schrödinger equation is the basic equation ruling the dynamics of a quantum system. However this equation is not relativistic (the kinetic energy is written as $\frac{\hbar^{2} k^{2}}{2 m}$ ) and a more general equation should be used (the Dirac equation). When expanding the Dirac equation to get relativistic corrections to the Schrödinger equation, a term which couples orbital and spin momenta appears. This Hamiltonian ${ }^{1}$ term is the spin-orbit coupling term $H_{s o}=\lambda \vec{l} . \vec{s}$. It can be seen as the coupling between the spin and the magnetic field created by the electron orbital current loop. For an electron in the spherical Coulomb potential of the atom nucleus (charge $\mathrm{Ze}$ ), $\lambda$ is proportional to $\frac{Z}{r^{3}}$. Heavy atoms such as rare-earths (large Z, smaller $r$, larger relativistic correction) have larger spin-orbit coupling constants $\lambda$ than lighter transition metals.

\section{ATOM MAGNETISM}

Magnetic atoms are multi-electron objects. Determining the fundamental electronic state of the atom is a complex many-body problem. Since only partially filled electronic shells contribute to spontaneous magnetic moments, filled shells are less relevant. It still leaves a complicated task to determine the most stable $3 \mathrm{~d}$ filling (10 quantum states) for $3 \mathrm{~d}$ transition metals and $4 \mathrm{f}$ filling (14 quantum states) for rare-earths. Once the electronic configuration is determined, the atom orbital and spin momenta can be calculated.

\subsection{Hund's phenomenological rules}

The fundamental electronic configuration for a subshell involves minimizing the energy which includes Coulomb interaction between the electrons and the nucleus but also electron-electron Coulomb repulsion and spin-orbit coupling. This has to be done respecting the Pauli principle (1925), i.e. one electron at most in each quantum state defined by its four quantum numbers $n_{i}, l_{i}, m_{i}, s_{i}$. Elaborate numerical calculations are possible but empirical Hund's rules allow a rapid determination of the likely fundamental configuration. One can then define the atomic spin moment $\mathrm{S}: \vec{S}=\sum_{i} \vec{s}_{i}$

Hund's $1^{\text {st }}$ rule states that $S$ should be maximum, taking the Pauli principle into account. It corresponds to minimizing Coulomb interaction between electrons.

\footnotetext{
${ }^{1} \mathrm{H}$ is the Hamiltonian or the magnetic field depending on paragraphs. B is always the magnetic induction field. S.I. units are always used.
} 


\section{Collection SFN}

After defining the atomic orbital momentum $\mathrm{L}$ as $\vec{L}=\sum_{i} \vec{l}_{i}$, Hund's $2^{\text {nd }}$ rule states that $\mathrm{L}$ should be maximum, respecting the Pauli principle and the first rule.

Once the spin and orbital moments are determined, the last rule helps to determine their relative directions. Hund's $3^{\text {rd }}$ rule couples $\vec{L}$ and $\vec{S}$, and it corresponds to using the sign of the spin-orbit coupling constant $\lambda$ in $H=\lambda \vec{L} \cdot \vec{S}$. It allows to calculate $\vec{J}=\vec{L}+\vec{S}$, the total angular momentum.

As a phenomenological rule, $\vec{L}$ and $\vec{S}$ are antiparallel, i.e. J $=|\mathrm{L}-\mathrm{S}|$ for a subshell less than halffilled and the coupling is parallel for more than half-filled subshells. The "half-filled" case is trivial since $\mathrm{L}=0$ for a half-filled subshell (gadolinium $4 \mathrm{f}^{7}$ for example).

For example, terbium is a rare-earth element. Let us define its stable electronic configuration according to Hund's rules. As a metal, in an alloy or in an oxide, terbium will always transfer 3 electrons to the conduction band or to the oxygen ion. The magnetic entity to consider is $T b^{+3}$, which has a $4 f^{8}$ partially-filled sub-shell. For a $4 \mathrm{f}$ sub-shell, $l=3$ and $s_{i}= \pm \frac{1}{2}$ so the $4 \mathrm{f}$ sub-shell has a degeneracy $2 \times 7=14$.

\begin{tabular}{|l|l|l|l|l|l|l|l|}
\hline$l_{i}$ & -3 & -2 & -1 & 0 & 1 & 2 & 3 \\
\hline$s_{i}=\frac{1}{2}$ & $\uparrow$ & $\uparrow$ & $\uparrow$ & $\uparrow$ & $\uparrow$ & $\uparrow$ & $\uparrow$ \\
\hline$s_{i}=\frac{1}{2}$ & $\downarrow$ & & & & & & \\
\hline
\end{tabular}

Following Hund's first rule, we find that the spin $S$ of terbium is: $S=\frac{7}{2}-\frac{1}{2}=3$. Its orbital momentum is $\mathrm{L}=3$ with $l_{i}=-3$ or +3 . Fortunately the $4 \mathrm{f}$ sub-shell is more than half-filled so its total angular momentum is $\mathrm{J}=\mathrm{L}+\mathrm{S}=6$. Some atoms lose magnetism due to the exact cancellation of $\mathrm{L}+\mathrm{S}$ !

\subsection{Atom magnetic moment}

As a straightforward consequence, the atom orbital magnetic moment is $\vec{m}_{L}=-\mu_{B} \vec{L}$ and the atom spin magnetic moment is $\vec{m}_{S}=-2 \mu_{B} \vec{S}$. The total atom magnetic moment is then $\vec{m}=-\mu_{B}(\vec{L}+2 \vec{S})$. The total atomic magnetic moment $\vec{m}$ is not proportional to $\vec{J}$ so in general not collinear with $\vec{J}$. It can be shown (Wigner-Eckart theorem) that the expectation value for the atomic magnetic moment is along its angular momentum. It is then possible to define the Landé factor $g_{J}$, which relates the total atomic magnetic moment and angular momentum along the quantification axis: $\langle\vec{m}\rangle=-g_{j} \mu_{B}\langle\vec{J}\rangle$.

$$
g_{J}=\frac{3}{2}+\frac{S(S+1)-L(L+1)}{2 J(J+1)}
$$

$g_{J}=1$ when $\mathrm{S}=0$ (pure orbital magnetism) and $g_{J}=2$ when $\mathrm{L}=0$ (pure spin magnetism). Possible values for the $(\mathrm{L}, \mathrm{S}, \mathrm{J})$ atom magnetic moment along $\mathrm{z}$ are $m_{J z}=-g_{J} M_{J} \mu_{B}$ with $M_{J} \in\{-J,+J\}$. Fully-filled subshells and empty subshells do not contribute to $\mathrm{S}$ and L. So the presence of partiallyfilled subshells is a requirement to get a spontaneous atomic magnetic moment.

As regards our previous example, the Landé factor for $T b^{+3}$ is $g_{J}=\frac{3}{2}+0=\frac{3}{2}$. So $T b^{+3}$ has a $9 \mu_{B}$ magnetic moment.

\subsection{Zeeman energy and diamagnetism}

The presence of a magnetic field will lift the $2 \mathrm{~J}+1$ degeneracy for $\vec{J}$. When an electromagnetic field is applied, the electron Hamiltonian has to be modified. To keep relativistic invariance, momentum $\vec{p}$ should be replaced by $\vec{p}-q \vec{A}$, where the vector potential $\vec{A}$ is given by $\vec{B}=c \vec{u} r \vec{A}$. Because of the differential operator $\overrightarrow{c u r l}$, a choice of gauge to define the vector potential $\vec{A}$ is possible. Let us look at the Schrödinger Hamiltonian first (spin-less electrons). $\mathrm{H}=\frac{p^{2}}{2 m}+V$ becomes $\mathrm{H}=\frac{1}{2 m}(\vec{p}-q \vec{A})^{2}+V$

In a uniform field $\vec{B}=B \vec{u}_{z}$, we can choose the vector potential $\vec{A}=\frac{1}{2} \vec{B} \wedge \vec{r}$. 
Expanding the square bracket, two new terms appear. The first one is the Zeeman Hamiltonian:

$$
\mathrm{H}_{\text {Zeeman }}=\frac{-q}{m} \vec{p} \cdot \vec{A}=\frac{e}{2 m}(\vec{r} \wedge \vec{p}) \cdot \vec{B}=-\vec{m}_{L} \cdot \vec{B} .
$$

This is the Zeeman effect on the orbital magnetic moment, it will lift the $\mathrm{J}$ degeneracy. Starting from the Dirac equation to make the spin appear, the complete Zeeman Hamiltonian comes as:

$$
\mathrm{H}_{\text {Zeeman }}=-\left(\vec{m}_{L}+\vec{m}_{S}\right) \cdot \vec{B} .
$$

The second term is called the diamagnetic Hamiltonian:

$$
\mathrm{H}_{\text {dia }}=\frac{q^{2}}{2 m} A^{2}=\frac{q^{2} B^{2}}{8 m}\left(x^{2}+y^{2}\right) .
$$

This diamagnetic Hamiltonian mixes applied field and the space coordinates. In order to make the magnetic moment $\vec{m}$ appear, it is possible to calculate it from $m_{i}=-\frac{\partial \mathrm{H}}{\partial B_{i}}$. For a uniform field along $\mathrm{z}$, one gets $\vec{m}=-\frac{e^{2}}{4 m} B_{z}\left(x^{2}+y^{2}\right) \vec{u}_{z}$.

The diamagnetic Hamiltonian shows that an applied magnetic field $\left(\vec{B}=\mu_{0} \vec{H}\right)$ induces a magnetic moment antiparallel to the field (negative susceptibility). The effect is proportional to the number of electrons and to the average orbit area. It is a temperature independent susceptibility. This diamagnetic moment is induced even in non magnetic materials such as silicon, quartz, $\mathrm{Cu}$ or $\mathrm{Bi}$. It is the main magnetic response of many non magnetic materials, especially non metals. For metals, the paramagnetic response from the conduction band counterbalances diamagnetism and the net susceptibility can have either signs.

\section{MAGNETIC MOMENT IN MATERIALS}

\subsection{Chemical bondings}

Up to now, the atom was supposed to be isolated, so within an isotropic potential. Magnetic materials are mostly in a solid state, so the magnetic atom is located in an atomic lattice with a perfectly long range order for crystals or with only short range order for amorphous and nanocrystallised materials. The impact of environment is very large. Looking at the Mendeleev table, most atoms have partially-filled subshells. So most of them (79 out of 103 elements) carry a magnetic moment when isolated (single atom gas).

In a solid, chemical bonds appear. The outer electronic subshells are deeply modified and they are, by definition, the partially filled subshells: He, Ar, Xe do not make chemical bonds. As a result of bonding, partially filled shells fill up and most elements lose their magnetic moment. Mainly two series of elements remain magnetic as a solid: $3 \mathrm{~d}$ transition metals and $4 \mathrm{f}$ rare-earths. Magnetism can also be found in other elements, which can become magnetic when alloyed e.g. $4 \mathrm{~d}(\mathrm{Pd}, \mathrm{Ru})$ and $5 \mathrm{~d}(\mathrm{Pt}, \mathrm{Re})$ transition metals or $5 \mathrm{f}(\mathrm{U})$ actinides.

\subsection{Crystal field}

For spheres of single-crystalline magnetic materials (isotropic shape), experimental measurement of magnetization, $\mathrm{M}(\mathrm{H})$ curves, show that most often, easy axes to magnetise the sample and harder axes may be distinguished. This anisotropy which comes from the crystalline nature of the material and not from its external shape is called the magneto-crystalline anisotropy. The origin is electrostatic. The first neighbor environment of each magnetic atom has an anisotropic electron density. The magnetic atom is under an anisotropic crystal electric field (CEF), so the orbital magnetism becomes anisotropic. Orbitals which were degenerate in an isotropic environment are split in this CEF. Since orbital and spin moments 


\section{Collection SFN}

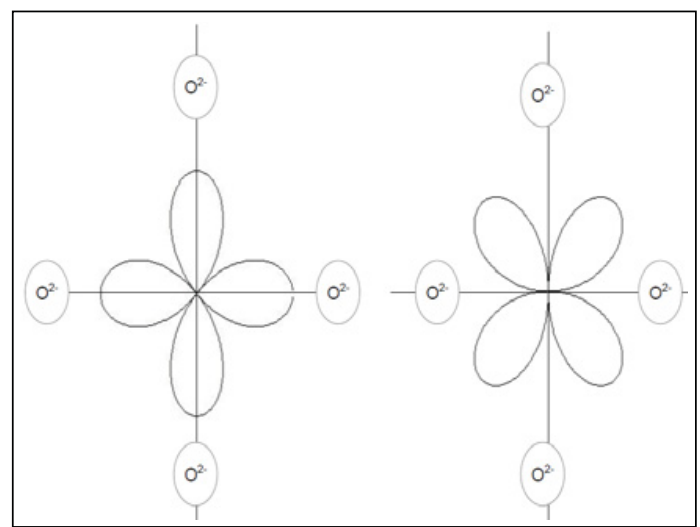

Figure 1. The crystal electric field from an oxygen octahedron environment lifts the degeneracy between $\mathrm{e}_{g}$ (left) and $\mathrm{t}_{2 g}$ (right) orbitals. $\mathrm{t}_{2 g}$ is favored.

are coupled through the spin-orbit coupling, the total magnetic moment lies preferably in directions called the easy axes.

The electron wavefunctions, described using spherical harmonics $Y_{l}^{m}(\theta, \phi)=A \cdot e^{i m \phi} P_{m}^{l}(\cos \theta)$, with $P_{l}^{m}$ Legendre polynoms, are the usual basis of eigen functions for a spherical environment. This is not the most suitable basis in the CEF environment. The local symmetry makes other bases a better choice to develop the wave functions. For example, in a cubic environment, for a $3 \mathrm{~d}$ subshell, a basis made out of two $e_{g}$ (also named $d_{z^{2}}$ and $d_{x^{2}-y^{2}}$ ) and three $t_{2 g}$ (also named $d_{x y}, d_{y z}$ and $d_{x z}$ ) functions corresponds to the doublet-triplet energy spectrum, once the 5 -fold degeneracy has been lifted by the CEF splitting energy.

$e_{g}$ and $t_{2 g}$ are built from linear combinations of spherical harmonics. For example, spherical harmonics $Y_{2}^{-2}(\theta, \phi)=A e^{-2 i \phi} \cdot \sin ^{2} \theta$ and $Y_{2}^{2}(\theta, \phi)=A e^{2 i \varphi} \cdot \sin ^{2} \theta$ can be replaced in a cubic environment by the following wavefunctions:

$e_{g}=d_{x^{2}-y^{2}}=\frac{1}{\sqrt{2}}\left(Y_{2}^{-2}+Y_{2}^{2}\right)=\frac{A}{\sqrt{2}} \cos (2 \phi) \cdot \sin ^{2} \theta$, its density mainly in the $x y$ plane pointing along $x$ and $y$ axes

and $t_{2 g}=d_{x y}=\frac{1}{\sqrt{2}}\left(Y_{2}^{2}-Y_{2}^{-2}\right)=\frac{A}{\sqrt{2}} \sin (2 \phi) \cdot \sin ^{2} \theta$, its density mainly in the $x y$ plane pointing $45^{\circ}$ away from $x$ and $y$ axes.

In an octahedric environment (for example a cation in an oxygen octahedron), the $t_{2 g}$ orbital will have a lower energy than the $e_{g}$ one since the $\cos (2 \phi)$ dependence brings the electron density close to the oxygen negative charges (Fig. 1).

The crystal field leads to a partial lift of the orbital degeneracy. It will also induce an orbital quench. In spherical coordinates the orbital moment operator is $L_{z}=\frac{\hbar \partial}{i \partial \phi}$. Applied to spherical harmonics it gives:

$$
\left\langle Y_{2}^{m}\left|L_{z}\right| Y_{2}^{m}\right\rangle=\left\langle Y_{2}^{m}|m \hbar| Y_{2}^{m}\right\rangle=m \hbar .
$$

So $Y_{2}^{m}$ is an eigen function for $L_{z}$ and its expected orbital moment is $m \hbar$.

Now what is the answer for $e_{g}$ and $t_{2 g}$ wavefunctions?

$\left\langle Y_{2}^{2}+Y_{2}^{-2}\left|L_{z}\right| Y_{2}^{2}+Y_{2}^{-2}\right\rangle=2 \hbar-2 \hbar=0$. We have used the orthogonality between $Y_{2}^{2}$ and $Y_{2}^{-2}$ functions.

The expected value for the orbital moment of the $e_{g}$ and $t_{2 g}$ orbitals is now zero. The orbital moment has been quenched by the CEF.

When considering the usual magnetic elements, rare-earths have a strong spin-orbit coupling and the CEF does not modify the electronic configuration. Moreover the $4 \mathrm{f}$ subshell is an inner shell which does 


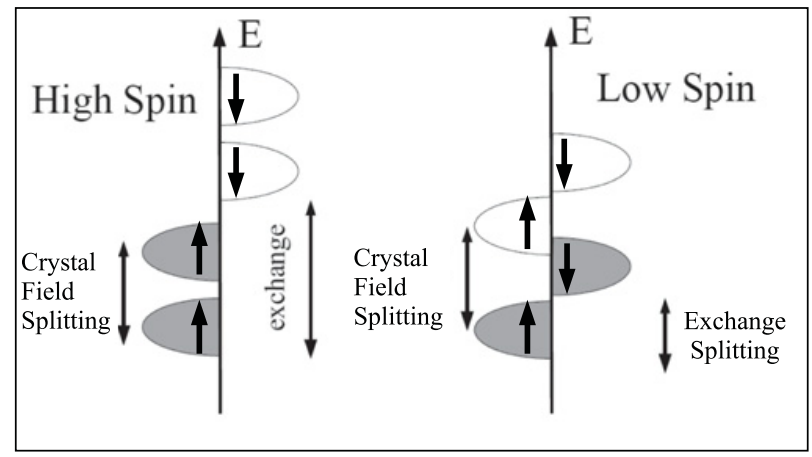

Figure 2. Depending on the relative amplitudes of the crystal electric field and exchange splittings, the atom may be in a high spin or low spin state.

not participate to chemical bondings ( $5 \mathrm{~d}$ and $6 \mathrm{~s}$ subshells do). The set of Hund's rules is a very good model for rare-earths. On the other hand, the CEF is usually large enough to quench the orbital moment of transition metal elements and only Hund's first rule applies, the orbital moment is much smaller than expected using the second rule.

When the CEF splitting energy is equivalent to the exchange splitting energy, high spin (Hund's first rule) and low spin (low lying CEF states filled first) configurations can be obtained (Fig. 2), sometimes including transition from one to the other when an external parameter (temperature, strain, field...) is varied.

\subsection{Magnetocrystalline anisotropy}

To describe the magneto-crystalline anisotropy, its energy can also be expanded using the spherical harmonics basis. The Crystal field Hamiltonian can also be rewritten in terms of $\mathbf{J}$ and $\mathbf{J}_{z}$ operators using Stevens operator equivalents.

From the experimental point of view, often not so many coefficients can be determined and simpler expressions involving incomplete and non orthogonal bases are used.

For materials with a single very easy axis, the magnetocrystalline anisotropy is modeled assuming a uniaxial symmetry as: $E_{M C}=K \sin ^{2} \theta$ When more data are available, a more complete model can be used:

$$
E_{M C}=K_{1} \sin ^{2} \theta+K_{2} \sin ^{4} \theta .
$$

In a hexagonal symmetry, it becomes: $E_{M C}=K_{1} \sin ^{2} \theta+K_{2} \sin ^{2} 3 \phi$.

In a cubic symmetry, $E_{M C}=K_{1}\left(\alpha_{1}^{2} \alpha_{2}^{2}+\alpha_{2}^{2} \alpha_{3}^{2}+\alpha_{3}^{2} \alpha_{1}^{2}\right)+K_{2} \alpha_{1}^{2} \alpha_{2}^{2} \alpha_{2}^{2}$ where $\alpha_{i}=\frac{1}{|\vec{m}|} \vec{m} \cdot \vec{u}_{i}$ are the 3 directional cosines defining the magnetic moment direction.

\section{PARAMAGNETISM}

When applying a magnetic field $\vec{H}$, one can define the response of a material by measuring or calculating its susceptibility $\chi$ defined as $\vec{M}=\chi \vec{H}$ or $\chi=\frac{\partial M}{\partial H} . \vec{M}$ is the magnetization i.e. the magnetic moment per unit volume (unit: $\mathrm{A} / \mathrm{m}$ ). Since the field unit is also $\mathrm{A} / \mathrm{m}, \chi$ is unitless. It can be a scalar or a tensor quantity according to the symmetry (or anisotropy) of the material.

It was already mentioned that even non magnetic (fully-filled subshells) materials have a small negative response (diamagnetism). The order of magnitude of the diamagnetic susceptibility is $\chi=$ $-10^{-6}$ (see Table 1$)$. 


\section{Collection SFN}

Table 1. Susceptibilities at $300 \mathrm{~K}$ for some diamagnets and paramagnets.

\begin{tabular}{|l|l|l|l|}
\hline Material & Susceptibility & Material & Susceptibility \\
\hline Water & $-910^{-6}$ & Bismuth & $-16510^{-6}$ \\
\hline Graphite HOPG $\chi_{\text {parallel }}$ & -400 to $-60010^{-6}$ & Copper & $-1210^{-6}$ \\
\hline Silicon & $-3.410^{-6}$ & Platinum & $+27810^{-6}$ \\
\hline
\end{tabular}

When no spontaneous (at zero field) magnetic order exists and when the susceptibility is positive, the material is a paramagnet. The paramagnetic state can be that of a magnetic material which never orders (non interacting moments). It is also the state of an ordered magnetic material, above its ordering temperature, when thermal excitations destroy the long range order. Paramagnetism can have several microscopic origins.

\subsubsection{Brillouin paramagnetism}

When existing moments are present at zero field but fluctuate in all directions because of thermal excitations, then the applied field serves to align the moments (minimising Zeeman energy) and to create a net magnetic moment. This is Brillouin paramagnetism and it is experimentally checked by measuring the susceptibility versus temperature. Since the order results from a competition between kT and Zeeman energy, $\chi$ follows the Curie law: $\chi=\frac{C}{T}$, where $\mathrm{C}$ is the Curie constant:

$C=\frac{\mu_{B}^{2}}{3 k_{B}} N g^{2} J(J+1)=\frac{m_{e f f}^{2}}{3 k_{B}} N$, with $\mathrm{N}$ the density of moments $/ \mathrm{m}^{3}, m_{e f f}=g \mu_{B} \sqrt{J(J+1)}$ is the effective moment i.e. the length of $\vec{m}$, longer that its maximum $m_{z}$ projection. At high fields or low temperatures, $\mathrm{M}(\mathrm{H})$ is not linear anymore. $\mathrm{M}(\mathrm{T}, \mathrm{H})$ follows a Brillouin function $B_{J}(x)$.

$$
M=M_{s a t} B_{J}(x) \text { with } M_{s a t}=N g_{J} J \mu_{B} \text { and } x=\frac{g_{J} J \mu_{0} \mu_{B} H}{k T}
$$

$x$ represents the ratio between Zeeman energy and thermal energy.

$$
\text { and the Brillouin function is } B_{J}(x)=\frac{2 J+1}{2 J} \operatorname{coth}\left(\frac{2 J+1}{2 J} x\right)-\frac{1}{2 J} \operatorname{coth} \frac{x}{2 J} \text {. }
$$

\subsubsection{Pauli paramagnetism}

In a metal such as platinum, no magnetic moment exists at zero field. When applying a magnetic field, the energy of conduction electrons will change by a quantity $\Delta E=-2 \mu_{B} \vec{s} \cdot \vec{B}$. An energy difference $2 \mu_{B} . B$ between the up spin and down spin bands appears. The response of the material is to transfer electrons from the higher energy band to the lower band. The number of electrons transferred is $\mu_{B} \cdot B \cdot N\left(E_{F}\right)$ with $\mathrm{N}$ the density of state at Fermi level. Each electron which flips increases the material magnetic moment by $2 \mu_{B}$. The susceptibility is then proportional to the density of state at the Fermi level: $\chi=2 \mu_{0} \mu_{B}^{2} N\left(E_{F}\right)$. Since the density of state of metals does not change much with temperature, unlike Brillouin susceptibility, Pauli susceptibility is quite temperature independent.

\section{INTERACTING MOMENTS}

In diluted magnetic materials, magnetic moments do not interact. The materials are paramagnetic. However when the density of magnetic moments increases, interactions appear. Several microscopic origins for interactions are possible. 


\subsection{Dipolar interaction}

Magnetic moments create a long range dipolar field around them. Any magnetic moment will then align in the resultant dipolar field. This is a long range interaction. To calculate the resulting field, the symmetry of the crystal has be to taken into consideration as well as the moment orientations with respect to this lattice. For a single moment $\vec{m}$, the dipolar field created by $\vec{m}$ can be written:

$$
\vec{H}_{\text {dipolar }}=\frac{1}{4 \pi}\left(\frac{3 \vec{r}(\vec{m} \cdot \vec{r})}{r^{5}}-\frac{\vec{m}}{r^{3}}\right) .
$$

At a distance of $0.2 \mathrm{~nm}$, a $1 \mu_{B}$ moment creates a maximum induction field of 0.2 Tesla. Summing over the complete lattice, the integrated dipolar field amplitude is of the order of 1 Tesla. For a $1 \mu_{B}$ moment, its Zeeman energy in a 1 Tesla field is equivalent to $\mathrm{k}_{B} \mathrm{~T}$ with $\mathrm{T}=0.6 \mathrm{~K}$. The consequence is that dipolar interaction may only be experimentally evidenced at very low temperatures, in the absence of larger interactions.

Since the dipolar field decays as $1 / r^{3}$, the dimensionality of the lattice matters. For example a 2D lattice of ferromagnetic dots can be considered as a 2D lattice of macrospins. Each macrospin creates a stray field which decays as $1 / r^{3}$. To evaluate the dipolar field on a specific site, one must sum up the contribution of all macrospins. Since the number of macrospins at a distance $r$ to $r+d r$ varies as $2 \pi r . d r$, their dipolar contribution scales as $\frac{2 \pi r \cdot d r}{r^{3}}=\frac{2 \pi . d r}{r^{2}}$. The contribution of far away spins decreases as $1 /$ r. The dipolar field loses its long range character for a 2D lattice. A large enough 2D lattice is not sensitive to its external shape unlike a 3D lattice, where the demagnetizing field and the induced shape anisotropy are given by the aspect ratio of the sample, even when its size is large compared to inter-atomic distances.

\subsection{Exchange interactions}

When a large density of magnetic moments exists, electronic wavefunctions of magnetic atoms start to overlap. The exchange path can be direct (first neighbor interaction) or indirect. For examples in oxides, since cations occupy interstitial sites in a compact oxygen lattice, cations first neighbors are oxygen ions. In magnetite $\left(\mathrm{Fe}_{3} \mathrm{O}_{4}\right) \mathrm{Fe}-\mathrm{Fe}$ exchange is indirect (it is superexchange interaction $\mathrm{Fe}-\mathrm{O}-\mathrm{Fe}$ ). In $\mathrm{Co} / \mathrm{Cu} / \mathrm{Co}$ multilayers, the exchange path is also indirect through the $\mathrm{Cu}$ conduction electrons. This exchange is oscillating with the Cu spacer thickness. It is the RKKY interaction named after Ruderman, Kittel, Kasuya and Yoshida.

The microscopic origin of the exchange interaction is once more electrostatic. When two magnetic atoms interact, since electrons can not occupy the same orbital if they have the same spin, parallel (ferromagnetic) configuration leads to occupation of different orbitals, decreasing the Coulomb repulsion which is modeled as $\mathrm{U}$ the on-site Coulomb energy: $H=U \sum n_{i \uparrow} n_{i \downarrow}$. However, occupation of different orbitals will lead to larger kinetic energy because the wavefunction is less delocalized across both neighbors. The balance, once again, is a many body problem, where electrons from the partiallyfilled bands and orbitals have to be taken into account as well as the lattice cell which may contain several non equivalent atoms.

Exchange between localized moments (in insulating oxides or in metallic $4 \mathrm{f}$ rare earth metals) will not be modeled using the same assumptions as the one used for metals such as iron or nickel where delocalized electrons require an itinerant modeling. Superexchange will require a precise description of the geometry in particular the angle cation-O-cation, as well as the nature and the filling of the orbitals involved in the exchange. Qualitative Goodenough-Kanamori rules allow to estimate the sign of the interaction and its strength. Exchange interaction in metals will require a description of the Fermi surface and nowadays can be obtained from ab-initio calculations. 


\section{Collection SFN}

Common models on a lattice include the Hubbard Hamiltonian:

$$
H=-\sum t_{i j} \cdot c_{i \sigma}^{+} c_{j \sigma}+U \sum n_{i \uparrow} n_{i \downarrow}
$$

with microscopic parameters $t$, the hopping integral and Coulomb repulsion $U$. Since $\Delta x . \Delta p>h$, larger hopping integrals mean more delocalized wavefunctions leading to a smaller kinetic energy. On a 3D lattice, with several orbitals per site and several sites per lattice cell, it is the source of infinite theoretical modeling. Such Hamiltonians based on microscopic ingredients $(t, U$, lattice) can be mapped on effective Hamiltonian such as the Heisenberg Hamiltonian: $H=J_{i j} \sum \vec{S}_{i} \cdot \vec{S}_{j}$. The directions of $\vec{S}$ can be fixed (Ising model) or allowed to change (XY or 3D). The lattice can be 1D, 2D, 3D. It can be geometrically frustrated (2D kagomé or 3D pyrochlore lattices). $J$ coupling can be isotropic or anisotropic and may involve only first neighbor or extends to second neighbors and farther.

\section{MAGNETIC ORDERS}

When magnetic moments interact, they do not always order. At high temperature, the magnetic state is always paramagnetic due to thermal excitation. When lowering the temperature, local order may appear. If long range order spontaneously appears (i.e. in the absence of an applied field) then a phase transition to a new magnetic state can be defined. If the moments order ferromagnetically, the transition temperature is called the Curie temperature. It is defined as the temperature for which a spontaneous magnetization appears. If moments order antiferromagnetically, no net magnetization appears. However, a long range order characterized by a well defined propagation vector is established and can be evidenced using neutron diffraction for example. The transition temperature is then called the Néel temperature.

Several antiferromagnetic orders are possible (antiferromagnetic coupling in all directions or only in one direction to result in antiparallely aligned ferromagnetic planes for example). Antiferromagnetic order of two non equivalent lattices may leave a net magnetization (ferrimagnetism). It is the configuration of many materials such as some rare-earth-transition metal alloys and compounds (GdCo) and many oxides, $\mathrm{Fe}_{3} \mathrm{O}_{4}$ (magnetite) or $\mathrm{Y}_{3} \mathrm{Fe}_{5} \mathrm{O}_{12}$ ( $\mathrm{YIG}$ garnet) for example. Non collinear orders may also exist (sperromagnetism (cone-like order), helicoïdal, cycloïdal orders...) and are associated to frustrated competing interactions or to anisotropic exchange interactions such as the DzyaloshinskyMoriya interaction in non centro-symmetric environments.

A mean field model can be often used to represent interactions in a simple manner. The microscopic details of interaction are replaced by an effective field proportional to the average magnetization of the relevant neighboring moments. This mean field or molecular field $\vec{H}_{m}=\lambda \cdot\langle S\rangle$ is then added to the applied magnetic field. Adding this molecular field is enough to induce a spontaneous magnetization in the Brillouin paramagnetic model. The order temperature for a simple ferromagnetic model is $T_{c}=\lambda C$.

$T_{c}$ is proportional to the mean field coefficient $\lambda$ and to the Curie constant $\mathrm{C}$. In the paramagnetic state (above $T_{c}$ ), the susceptibility follows a modified Curie law called the Curie-Weiss law: $\chi=$ $\frac{C}{T-T_{c}}$. Without interaction $T_{c}=0$. With negative (antiferromagnetic) interactions, the Curie-Weiss law becomes $\chi=\frac{C}{T+T_{N}} . T_{c}$ and $T_{N}$ determined using a Curie-Weiss law are sometimes identified to the Curie temperature and the Néel temperature. This is not the correct definition for these transition temperatures which should be defined from the onset of long range magnetic order. $T_{c}$ and $T_{N}$ determined from a Curie-Weiss law should be called the paramagnetic Curie temperature and the paramagnetic Néel temperature and can be named $\theta_{c}$ and $\theta_{N}$. For practical purposes, plotting the inverse of the susceptibility as a function of temperature, allows to evidence the presence of ferromagnetic or antiferromagnetic interaction. When the Curie-Weiss law stands, it also allows to estimate the effective magnetic moment from the Curie constant. 


\section{EXCITATIONS AND DYNAMICS}

Magnetic excitations can disorder the magnetic saturated state (similar to phonon disordering the periodic crystal lattice leading to its melting). These excitations can be modeled as spin waves and quantized as magnons. They can be measured using inelastic neutron scattering. Similar to phonon spectra, magnetic excitations spectra allow to define branches. Unlike phonons which have as a first approximation a linear dispersion curve $\hbar \omega=D q$, with $q$ the excitation wavevector, spin waves in a ferromagnet have a quadratic $q$-dependence $\hbar \omega=D q^{2}$, with a possible gap because of magnetic anisotropy. In an antiferromagnet, the spin wave dispersion recovers a linear $q$-dependence. Thermal excitation of spin waves in a ferromagnet leads to a temperature decrease of the magnetization called the Bloch $\mathrm{T}^{3 / 2}$ law: $M(T)=M(0 K)\left(1-a T^{3 / 2}\right)$, valid far below $\mathrm{T}_{c}$.

Dynamics of magnetism spans orders of magnitude ranging from ultrafast demagnetization at the femtosecond scale, to magnetization precession at the nanosecond scale up to domain wall propagation with velocities smaller than sound waves (typically $1 \mathrm{~km} / \mathrm{s}$ in a solid). Electron processes are associated to typical energies of $1 \mathrm{eV}$ i.e. to a frequency $E=h v \approx 2.10^{14} \mathrm{~Hz}$ or a time scale of a few femtoseconds. Thermalisation of the electronic distribution happens at the femtosecond scale but thermalisation with the lattice happens at a much slower timescale (picosecond). Magnetic precession happens for a well defined magnetic moment in thermal equilibrium. Its time scale is related to Larmor precession, which can be calculated from the dynamics of the magnetic moment. It can also be recovered from the dynamics of the angular momentum. Since the atom angular momentum is $\vec{\sigma}=\hbar \vec{J}$ and the atom magnetic moment is $\vec{m}=-g_{J} \mu_{B} \vec{J}$, their ratio is called the gyromagnetic factor $\gamma$ :

$$
\gamma=\frac{m}{\sigma}=-\frac{g_{J} \mu_{B}}{\hbar}=-\frac{g_{J} e}{2 m} .
$$

Considering the torque experienced by a magnetic moment in an applied field, the dynamics of its angular momentum is:

$$
\frac{d \vec{\sigma}}{d t}=\vec{m} \times \mu_{0} \vec{H} \text { i.e. } \frac{d \vec{m}}{d t}=\gamma \vec{m} \times \mu_{0} \vec{H} .
$$

This precessional motion can also be seen from the Zeeman splitting associated to the applied field. In a magnetic induction $\mathrm{B}$, the degeneracy of a spin $1 / 2$ energy levels is lifted. A spin $1 / 2$ can absorb a photon with frequency $v: h v=g \mu_{B} B$. For most materials $g$ value is close to $2 . g=2$ gives $\gamma=1.7610^{11} \mathrm{~Hz} / \mathrm{T}$. In a 1 Tesla field, the Larmor precession frequency is $28.04 \mathrm{GHz}$, i.e. a sub-nanosecond timescale.

In a material, the Zeeman energy is not the only energy which induces a torque on the magnetic moment. All energy terms can induce torques and the applied magnetic field has to be replaced by an effective field defined as:

$$
\vec{H}_{e f f}=-\frac{1}{\mu_{0}} \frac{\partial E}{\partial \vec{M}}
$$

The effective field includes contributions from the applied field (Zeeman energy), the demagnetizing field (shape anisotropy) and also magnetocrystalline and exchange energies.

In a real system, the precession of the magnetic moment is dampened, so that the moments align with the field. A phenomenological damping coefficient $\alpha$ is included and gives the Landau-Lifschitz equation, which is commonly used to describe magnetic moment dynamics. The magnetization $\vec{M}$ is used instead of the atomic moment $\vec{m}$.

$$
\frac{d \vec{M}}{d t}=\gamma \vec{M} \times \mu_{0} \vec{H}_{e f f}+\alpha \vec{M} \times\left(\vec{M} \times \vec{H}_{e f f}\right)
$$

Mathematically, it can be rewritten as the equivalent Landau-Lifschitz-Gilbert (LLG) equation:

$$
\frac{d \vec{M}}{d t}=\gamma \vec{M} \times \mu_{0} \vec{H}_{e f f}+\alpha^{\prime} \vec{M} \times\left(\frac{d \vec{M}}{d t}\right) .
$$




\section{Collection SFN}

Table 2. Magnetic properties for some common ferromagnets.

\begin{tabular}{|c|c|c|c|c|}
\hline Material & $\begin{array}{c}\text { Magnetocrystalline } \\
\text { Anisotropy K }\left(\mathrm{J} / \mathrm{m}^{3}\right)\end{array}$ & $\begin{array}{c}\text { Exchange constant } \\
\mathrm{A}(\mathrm{pJ} / \mathrm{m})\end{array}$ & $\begin{array}{c}\text { Domain wall energy } \\
\gamma\left(\mathrm{J} / \mathrm{m}^{2}\right)\end{array}$ & $\begin{array}{c}\text { domain wall width } \\
\delta(\mathrm{nm})\end{array}$ \\
\hline Fer & $510^{4}$ & 8 & 2.5 & 40 \\
\hline Cobalt & $510^{5}$ & 10 & 8.9 & 14 \\
\hline Nickel & $510^{3}$ & 3 & 0.5 & 37 \\
\hline $\mathrm{Nd}_{2} \mathrm{Fe}_{14} \mathrm{~B}$ & $510^{6}$ & 7 & 24 & 3.7 \\
\hline
\end{tabular}

\section{MAGNETIC PROCESSES}

In order to calculate the magnetic field created by a magnetic material (stray field outside and demagnetising field inside), an electrostatic analogy, allows to correctly describe the magnet as magnetic charges. Volume charges are given by $-\operatorname{div} \vec{M}$ (none when the magnetization is uniform) and surface charges by $\vec{M} \cdot \vec{n}$, with $\vec{n}$ the unit vector perpendicular to the surface, outward oriented. Stray fields around the magnet are created by these magnetic charges (or poles). The net charge is zero since a magnetic moment is a dipole. These charges are fictitious.

In a magnetic material with non-zero spontaneous magnetization, shape anisotropy will force the magnet to minimize the quantity of magnetic charges. In a first step, magnetization aligns along the long axis of the material. If charges are still present and create stray fields, the material will then demagnetize. Demagnetizing a sample means creating magnetic domains. In a domain, magnetization is uniform. Between domains, domain walls represent a transition zone where the magnetization is not uniform (exchange energy cost) and it is not align with magnetic easy axes (magnetocrystalline energy cost). A balance has to be made to reduce the magnetization and the associated demagnetizing energy, not increasing too much the cost due to domain walls. The energy minimum gives the optimum domain size, as well as the optimum domain wall position, thickness and internal structure (magnetization configuration). Calculating this balance is one aim of micromagnetism.

Micromagnetism's main assumption is that the local magnetization amplitude is constant, only its direction has to be calculated. Most experimental systems contain too many atoms for an atomic calculation to be possible and continuous model are usually considered. Heisenberg Hamiltonian can be mapped onto a continuous exchange interaction characterized by the exchange constant $\mathrm{A}(\mathrm{J} / \mathrm{m})$, see Table 2 for some values. The exchange energy cost (compared to uniform magnetization) can be written as a function of the space derivatives of the magnetization components:

$$
\text { Micromagnetism exchange energy }\left(\mathrm{J} / \mathrm{m}^{3}\right)=A \sum_{x, y, z}\left(\nabla \frac{M_{i}}{M_{s}}\right)^{2} .
$$

Associated to the demagnetizing energy $E_{d}=-\frac{\mu_{0}}{2} \vec{M} \cdot \vec{H}_{d}$, it allows to define the exchange length $l_{e x}$, i.e. the distance below which the magnetization can be considered to be uniform.

$$
l_{e x}=\sqrt{\frac{2 A}{\mu_{0} M^{2}}} .
$$

A typical value for the exchange length is $l_{e x}=3 \mathrm{~nm}$. It also gives the minimum size for meshing a magnetic material in a finite element model.

Comparing exchange energy and magneto-crystalline energy $E_{M C}=K \sin ^{2} \theta$, with $K$ the magnetocrystalline anisotropy constant for a uniaxial anisotropy and $\theta$ the angle between magnetization and easy axis, one also gets the domain wall width $\delta$ and domain wall energy $\gamma$ see Table 2

$$
\delta(\mathrm{m})=\pi \sqrt{\frac{A}{K}} \text { and } \gamma\left(\mathrm{J} / \mathrm{m}^{2}\right)=4 \sqrt{A K} \text { for a } 180^{\circ} \text { Bloch wall. }
$$


So for a Bloch wall (magnetization $180^{\circ}$ rotation, keeping $\vec{M}$ parallel to the wall plane, $\operatorname{div} \vec{M}=0$ i.e. no volume charge in the wall), a large magnetocrystalline anisotropy corresponds to a narrow domain wall (permanent magnets) and a small anisotropy corresponds to a wide domain wall (soft magnets). For a thin film with in-plane magnetization, a Bloch wall would induce out-of-plane magnetization i.e. demagnetizing field and it is therefore not favored. A phase diagram (wall configuration - film thickness) can be determined, and many other wall configurations are found (Néel walls, transverse walls, vortex walls in stripes...)

Size permitting, closure domains may appear to prevent surface charges i.e. allowing to keep $\vec{M}$ parallel to the surface of the sample.

\section{MAGNETIZATION REVERSAL}

The energy that needs to be minimized to get the stable configuration of a magnet is the sum of four terms. The exchange term aims at a uniform magnetization. The Zeeman term tends to align magnetization and applied field. The demagnetizing term tends to minimize the demagnetizing field, i.e. to align the magnetization parallel to the surface without bulk divergence or to demagnetize the sample. The last term forces the magnetization to align with magnetocrystalline anisotropy easy axes. The optimum configuration is usually a balance between competing energies.

In order to reach its optimum magnetic configuration, a sample needs to modify its magnetization. However, magnetic processes are not reversible and the optimum configuration is not always obtained. Studying magnetization reversal and $\mathrm{M}(\mathrm{H})$ loops, one can define the coercive field $H_{c}$, which is the field required to reverse $50 \%$ of $\mathrm{M}$ (i.e. reaching zero net magnetization) and the remanent magnetization (magnetization at zero field after saturation). In order to reverse magnetization, nucleation of antiparallel domains and propagation of domain walls are two possible mechanisms.

When the sample size is smaller than the domain wall width, magnetization reversal takes place without domains. Uniform rotation of the magnetization takes place for the smallest particles, and is adequately modeled using the Stoner-Wolhfahrt equation i.e. minimizing $E=E_{\text {Zeeman }}+E_{\text {demag }}+$ $E_{\text {anisotropy }}$ (no exchange energy, the magnetization stays uniform during reversal). Very small particles can reverse but can not be demagnetized (radius below the single domain critical size). Small material volume also means that intrinsic properties evolve. Surface anisotropy may appear due to reduced symmetry of the environment for surface atoms. Surface anisotropy may dominate the bulk magnetocrystalline anisotropy for nanometer-thick films or particles. Surface environment may also modify orbital moment quenching and orbital magnetic moments may drastically vary close to the surface.

\section{BASIC EQUATIONS AND UNITS}

\section{1 magnetic field and induction field}

In a magnetic material, great care has to be taken to distinguish:

$\vec{B}$ : the magnetic induction field (in Tesla) and $\vec{H}$ : the magnetic field (in A/m).

They are always related through the state equation: $\vec{B}=\mu_{0}(\vec{H}+\vec{M})$, with $\mu_{0}=4 . \pi \cdot 10^{-7}$ S.I. but $\vec{B}$ and $\vec{H}$ are not anymore systematically collinear in a magnetic material when $\mathrm{M}$ is not zero.

The magnetic field $\vec{H}$ in a material is always the sum of two contributions:

$$
\vec{H}=\vec{H}_{\text {applied }}+\vec{H}_{\text {demag }} .
$$

Zero applied field and zero field do not always happen at the same time. In particular remanence is experimentally easily obtained at zero applied field but a demagnetizing field is often still present. 


\section{Collection SFN}

Spontaneous magnetization used to define the Curie temperature is a zero field extrapolate and not a zero applied field extrapolate.

The sample magnetic moment $\vec{m}$ (in $\mathrm{Am}^{2}$ ) is related to the magnetization $\vec{M}$

using $M=\frac{\text { magnetic moment }}{\text { unit volume }}$ (in $\mathrm{A} / \mathrm{m}$ ).

\subsection{Maxwell equations}

Maxwell equations in a magnetic material:

$\operatorname{div} \vec{B}=0$

$\vec{c}$ url $\vec{H}=\vec{j}+\frac{\partial \vec{D}}{\partial t}$.

\section{3 c.g.s. system}

S.I. vs c.g.s units

Induction Field: 1 Tesla $=10000$ Gauss

in vacuum, $\mathrm{B}=1$ Gauss corresponds to $\mathrm{H}=1$ Oersted, unfortunately $\mu_{0}=1$ in cgs units, which is the reason why cgs is still popular and it does not help to distinguish $\vec{B}$ and $\vec{H}$, especially in air where they have the same numerical values in c.g.s.

In S.I. units, the sum of the three demagnetizing coefficients is 1 . This sum is $4 \pi$ using cgs.

$\vec{B}=\mu_{0}(\vec{H}+\vec{M})$ becomes in cgs $\vec{B}=\vec{H}+4 \pi \vec{M}$.

The magnetic moment unit in cgs is the emu (electro-magnetic unit) with: $1 \mathrm{emu}=10^{-3} \mathrm{Am}^{2}$.

The moment per unit mass produces a simple equivalence: $1 \mathrm{Am}^{2} / \mathrm{kg}$ is $1 \mathrm{emu} / \mathrm{g}$.

\section{References}

"Permanent Magnets", R. Skomski, J.M.D. Coey, IoP publishing (1999).

"Magnetism and Magnetic Materials", J.M.D. Coey, Cambridge Univ. Press (2010).

"Modern Magnetic Materials: Principles and Applications", Robert C. O’Handley, Wiley (2000).

“Simple Models of Magnetism”, R. Skomski, Oxford Univ. Press (2012). 